\title{
KEY ASPECTS OF THE PROPER FORMULATION OF THE MODEL IN NUMERICAL ANALYSIS OF THE INFLUENCE OF MINING EXPLOITATION ON BUILDINGS
}

\author{
LUCYNA FLORKOWSKA, JAN WALASZCZYK
}

The Strata Mechanics Research Institute of the Polish Academy of Sciences, ul. W. Reymonta 27, 30-059 Kraków, Poland, e-mail: florko@img-pan.krakow.pl

\begin{abstract}
Numerical modelling is an important tool used to analyse various aspects of the impact of underground mining on existing and planned buildings. The interaction between the building and the soil is a complex matter and in many cases a numerical simulation is the only way of making calculations which will take into consideration the co-existence of a number of factors which have a significant influence on the solution. The complexity of the matter also makes it a difficult task to elaborate a proper mathematical model - it requires both a thorough knowledge of geologic conditions of the subsoil and the structural characteristics of the building.

This paper discusses the most important problems related to the construction of a mathematical model of a building-mining subsoil system. These problems have been collected on the basis of many years of experience the authors have in observing the surveying and tensometric deformations of the rock-mass and buildings as well as in mathematical and numerical modelling of the observed processes.
\end{abstract}

Key words: numerical modelling, building, subsoil, mining, impact of mining exploitation on buildings

\section{INTRODUCTION}

Scientific research on the impact of mining on land surface and buildings is carried out in the context of using its findings to minimise the adverse effects of excavation. These effects have an environmental dimension as well as economic and social ones [1], [3]. The impact of underground mining leads to land surface degradation, altered geomorphology of the area, distorted hydrology and changes to local ecosystems. It causes damage to and destruction of technical infrastructure elements such as buildings, roads, service infrastructure networks and it decreases the quality of life of the residents.

Recognising and understanding the phenomena caused by underground mineral mining as well as their impact on the subsoil and buildings is a fundamental problem in the entire area of activity collectively termed as mining areas protection. For the above reason this research is of paramount importance. Its proper conduction, the selection of appropriate methods, procedures and research tools are the conditions which warrant the reliability of the findings, which after all are the basis for all the preventive measures, both in the area of mining (appropriate manner of conducting the exploitation) and in the area of construction (suitable safety measures for buildings).

One of the tools of cognition mentioned by modern philosophy of science, apart from experiment (observation) and idealisation (choosing the essential properties of things), is mathematical modelling. When formulating a problem, modern science searches for a solution to it using all the resources that mathematics has at its disposal - the most powerful and the most objective cognition tool we possess. This approach is used especially in technical sciences, where research on mathematical models is used. The next stage in this type of research is validating and evaluating it on material models and on real structures. Mathematical modelling permits the cost of research to be optimised but also to analyse processes at a level inaccessible to direct observation. There are many areas of science in which research the mathematical model, verified only for subsequent particular cases, is the only method of observing and trying to understand the reality.

The impact of underground mining on buildings is one of those problems in which analysing the mathematical model is the only method available to carry 
out an analysis which will comprise all the most important factors influencing the solution [3]. However, elaborating a proper mathematical model, calibrating and verifying it is a complex and challenging task. The most important aspects and problems connected with this matter shall be discussed in this article.

\section{FORMULATING THE PROBLEM}

In the analysis of the impact of underground mining on a building a complex system consisting of the building and its subsoil is under review. The subsoil can be imagined as a cut-out fragment of a rock mass with the dimensions selected in such a manner that the impact of the building on the state of stress would be negligibly small near the boundary of the fragment. The building is connected with the subsoil through the foundations sunk into it, which results in an interaction between them.

The rock mass around the building is deformed as a result of underground mining exploitation. The deformations of the subsoil change the initial state of stresses around the foundations and via the foundations the impact is transferred to the entire structure of the building. The whole process takes place in space and time.

Since the mining exploitation are carried out at considerable depths in relation to the building size, the model of the problem comprising the process of mining would be very large. For this reason a solution is used which consists in replacing mining with its impact, expressed as boundary conditions which change over time and in space. Depending on the type of analysis, these conditions can be calculated on the basis of the deformation model adopted or on the basis of measurement results.

The most important characteristics of the problem under review can be summarised as follows [2]:

- The system is composed of two elements: the building and the subsoil which interact with each other; the building transfers all the loads connected with its dead load and its environmental and functional loads to the subsoil and the subsoil transfers mining-induced deformations to the building.

- The subsoil is a much more flexible medium than the foundations and the building; its behaviour is definitely non-elastic and is connected with large (finite) deformations.
- The interactions taking place in the contact area are transferred in the normal direction to the contact surface and, through friction in the tangent direction, slides can occur in the contact area.

The problem thus formulated requires a mathematical model which will describe the behaviour of the system in the most accurate way possible.

\section{OBSERVATION - THE SOURCE OF KNOWLEDGE ABOUT THE MODELLED STRUCTURE}

Reliable knowledge about the modelled structures is fundamental for elaborating an appropriate model. The results of observation (measurements) are the main source of this knowledge. In scientific research on the impact of mining on buildings both material objects and processes and phenomena are observed. The first category includes: the building together with the subsoil and the existing protection system [4]. The second category includes displacement processes taking place in both the subsoil and the building and processes taking place at the subsoil-foundation interface and responding to the transfer of interactions between the building and the subsoil.

The observation is carried out using measuring equipment and devices as well as the senses, with each of these methods being of essential significance. The measurement systems installed both on the structure and in the subsoil provide a continuous observation of the deformation process and can be used to continuously monitor the condition of the structure. The important drawback here is the point nature of this type of observations. This means that the results of such observations do not provide an image of how the entire system under review behaves. The part of the structure which is outside the area covered by the measuring equipment sensors remains "invisible" for this kind of system [5], [10]. For this reason, the most frequently used and, so far, the most reliable observation method is continuous technical supervision consisting in regular checks of the structure performed by persons with relevant knowledge and experience [2].

\section{IDEALISATION}

Elaborating the model is connected with identifying the processes and phenomena which have an es- 
sential significance for the problems under review. Thus, idealisation is performed, which consists in identifying the essential characteristics and characteristics which can be neglected.

In the case of modelling the impact of underground mining on buildings the following are the factors which will determine the solution in the most significant manner: the mining situation and history as well as the geologic structure - for the subsoil; the geometry, the type of construction and foundations as well as the age and technical condition - for the building. Therefore, the mathematical model which will be the basis for the analysis should make it possible to include the characteristics of the problem listed above. It is also important to note that the adopted mathematical description should permit the necessary parameters to be reliably determined. The model which precisely describes the behaviour of a building and subsoil becomes useless in a situation when no practical possibilities exist of conducting the analyses necessary to calibrate it properly.

\section{THE MATHEMATICAL DESCRIPTION OF THE PROBLEM}

Let us now consider the problem described in the previous subchapters of using continuous media mechanics methods. It will be tantamount to making an assumption that the system under review is a material continuum. The problem then belongs to the category of contact problems and can be in general a non-linear problem, where both a physical nonlinearity related to the behaviour of the material (mainly the subsoil) and a geometric non-linearity caused by the existence of finite strain in the medium exist [2].

Formulating the set of equations we shall apply a material description in the three-dimensional, Cartesian coordinate system $x_{i}=\left\{x_{1}, x_{2}, x_{3}\right\}$. Due to large deformations in the subsoil medium the Green-de Saint Venant strain tensor $E$, which expresses the total strain in the medium in relation to the initial configuration, shall be the measure of the strain

$$
\begin{gathered}
\boldsymbol{E}=\frac{1}{2}(\boldsymbol{C}-\boldsymbol{I}), \\
E_{i j}=\frac{1}{2}\left(C_{i j}-\delta_{i j}\right),
\end{gathered}
$$

where $C$ is the Cauchy strain tensor

$$
\begin{aligned}
\boldsymbol{C} & =\boldsymbol{F}^{T} \boldsymbol{F}, \\
C_{i j} & =F_{k i} F_{k j},
\end{aligned}
$$

$\boldsymbol{F}$ is the deformation gradient.

The nominal stress tensor (the first Piola-Kirchoff tensor) $\boldsymbol{S}$ will be the stress tensor

$$
\boldsymbol{S}=J \boldsymbol{F}^{-1} \boldsymbol{\sigma}
$$

where

$\mathrm{J}=\operatorname{det} \boldsymbol{F}$,

$\boldsymbol{F}$ - the deformation gradient,

$\sigma-$ Cauchy stress tensor.

The set of equations for the problem of the interactions between the building and the subsoil is as follows:

- equilibrium equations:

$$
S_{i j, j}+\rho_{0} b_{i}=\rho_{0} \ddot{u},
$$

$\rho_{0}-$ initial mass density,

$b_{i}-$ body forces;

- geometric equations:

$$
E_{i j}=\frac{1}{2}\left(u_{i, j}+u_{j, i}+u_{k, i} u_{k, j}\right) \text {; }
$$

- constitutive laws expressing the relationship between the measure of the stresses and the measure of the strains.

In the problems of mechanics the constitutive laws are given by the relationships between the state of stress in the medium and the state of its strain. The still developing theory of constitutive equations provides more and more extensive laws describing various behaviours of materials. The choice of an appropriate material law is directly connected with analysing the character and properties of a given medium, which, in turn, is based on laboratory and in-situ tests. A vast majority of research on the properties of materials is carried out in laboratories where the right conditions are created to make observations and measurements determining the state of stress-strain relationship of the medium as well as the relationships between stress/strain and strain velocity, temperature or other fields of variables. Analysing the criteria which are decisive for a change in the behaviour of the medium, for the transition between the elastic and plastic, viscous or brittle behaviours is a very important problem in mechanics. Looking for the shapes of transition surface between the different behaviour phases of materials or for the failure surface in the stress space requires laboratory experiments with the 
use of specialist equipment able to create complex states of stress in the material.

\section{INITIAL BOUNDARY CONDITIONS AND CONTACT CONDITIONS}

- The kinematic boundary conditions on the nonloaded boundary:

$$
S_{i j} n_{j}=0 .
$$

- The kinematic displacement boundary conditions in the initial configuration (for $t=0$ ) on the boundary of the subsoil block:

$$
\begin{aligned}
& u_{1}=0, \\
& u_{2}=0, \\
& u_{3}=0,
\end{aligned}
$$

where $u$ is the displacement vector.

- The kinematic displacement boundary conditions in the present configuration (for $t>0$ ):

$$
u_{i}=f\left(x_{i}, t\right) \quad \text { on } \quad \Gamma_{G u} \forall t>0
$$

where $\Gamma_{G u}$ is the fragment of the subsoil boundary where displacement boundary conditions are set.

The displacements on the $\Gamma_{G u}$ boundary simulate the subsoil deformations due to mining. As has already been said, they can be set as:

- The Budryk-Knothe theory equation [8], [9].

In the analysis predicting the state of a structure the parameters of constitutive equation used can then be determined on the basis of the shapes of mining troughs which are formed in a given area during past exploitations or on the basis of the knowledge found in literature.

In the analysis of the already finished mining exploitation the parameters of the theory should be determined on the basis of the shape of the trough formed as a result of mining of a given longwall (or longwalls).

- The equation obtained from the approximation of the surveying measurement results.

Contact conditions:

- normal contact:

$$
\boldsymbol{T}_{n}=(\boldsymbol{S n}) \boldsymbol{n}
$$

where

$\boldsymbol{T}$ - the nominal contact stress tensor,

$\boldsymbol{T}_{\boldsymbol{n}}-$ the normal nominal contact stress tensor,

$\boldsymbol{n}$ - the vector normal to the surface of the contact;

- tangent contact:

$$
\begin{array}{ll}
\left|\boldsymbol{T}_{s}\right|<f \boldsymbol{T}_{n}, & \dot{\boldsymbol{u}}_{T}=0, \\
\left|\boldsymbol{T}_{s}\right|=f \boldsymbol{T}_{n}, & \dot{\boldsymbol{u}}_{T}=-\lambda \boldsymbol{T}_{s},
\end{array}
$$

$$
\boldsymbol{T}_{s}=\boldsymbol{T}-\boldsymbol{T}_{n},
$$

$\boldsymbol{T}_{\boldsymbol{s}}$ - the tangent nominal contact stress tensor,

$s$ - the vector tangent to the surface of the contact,

$f$ - friction coefficient.

\section{THE VIRTUAL WORK PRINCIPLE}

The Finite Element Method shall be used to find a solution to thus formulated problem. In this case, the virtual work principle shall be the fundamental equation

$$
\int_{\Gamma} p_{i} u_{i} d S+\int_{\Omega} b_{i} u_{i} d \Omega=\int_{\Omega} \sigma_{i j} \varepsilon_{i j} d \Omega
$$

where

$p_{i}-$ surface forces,

$b_{i}-$ body forces.

The above system of equations is a general model of the problem of the interaction between the building and the mining subsoil undergoing deformation. It can be brought to a specific form for concrete cases under consideration. However, it is extremely important to correctly prepare the collection of input data, which will be used to specify the geometry, the constraints and the form and parameters of assumed equations.

\section{DETERMINING INPUT DATA FOR CORRECT MODELLING}

The necessary condition to perform a correct analysis is to recognise the geologic and mining situation, to assess the construction conditions, to identify the kinematic constraints and load conditions as well as to identify all the factors which might influence the state of the subsoil-building system in a significant way. This analysis is carried out on the basis of the available technical documentation of the building, quantity surveys and site inspections, the data about the mining situation and history and the information about the subsoil. The available documents, designs, expert opinions and measurement documentation from a given area should be reviewed as extensively as possible and the obtained information should be analysed. With existing structures, especially old buildings, a common problem is missing or incomplete technical documentation of the building. If the missing documentation cannot be restored, surveying is often the only solution left (which is quite inconvenient in the case of inhabited buildings). 
The second group of data is the material obtained through tests and measurements. The results of measuring the deformations of the surface and the buildings play an important role. Most frequently these are the results of surveying measurements carried out by mining company surveyors. The collections of regular measurements carried out by these surveyors are an important source of information about the behaviour of the rock mass in the given area and combined with the maps of longwall panel development over time and maps of areas mined may be the basis for determining the real deformation indicators in the analysed area. These results are often of little use, however, for the analyses of buildings due to the low frequency of making the measurements.

It is best to determine the data about the mechanical properties of the geological materials making up the subsoil and building materials in laboratory tests or in situ. In the case of a planned building the information about the material properties included in the technical documentation can be used. When conducting an analysis of a historical building changed as a result of many years of exposure to various environmental factors and made of degraded material, it is advisable to obtain samples and carry out laboratory tests. Unfortunately, it is often impossible to obtain a sufficient number of samples and in the right shape to determine the parameters for an advanced material model, e.g., a historical wall. In such cases, what remains is to adopt a simple physical law and evaluate the material properties on the basis of the analysis of small samples and the approximation of the results using mathematical modelling.

The following are the most important groups of input data for the model:

1. The geologic and geotechnical recognition is the basis for the formulation of the initial conditions reflecting the state of primary geostatic stresses in the subsoil, the contact conditions between the ground and the foundations and for establishing the structure of the geometric area in the model. In order to define the geometric area of the problem data about the geometry of the building and the subsoil must be entered. As far as the subsoil geometry is concerned, the shape dimensions are chosen in such a way that beyond them the impact of the building should be minimal. Whether this effect has been achieved should be verified at the stage of making preliminary calculations. The division of the subsoil into individual geotechnical layers is performed on the basis of a geologic analysis [1]. If data concerning this aspect are absent in the existing documentation, exploratory boreholes should be made in order to obtain the geologic profile of the subsoil and to collect samples for laboratory tests. Figures 1 and 2 shows the photographic documentation of samples taken from the boreholes made during the initial analyzes of impact of the planned excavation on the building located in Ruda Śląska (Silesia, Poland) and the basic parameters of the Mohr-Coulomb material law determined on the basis of laboratory tests for geotechnical clay layer (depth: $-1.5 \div-2.9 \mathrm{~m})[2]$.

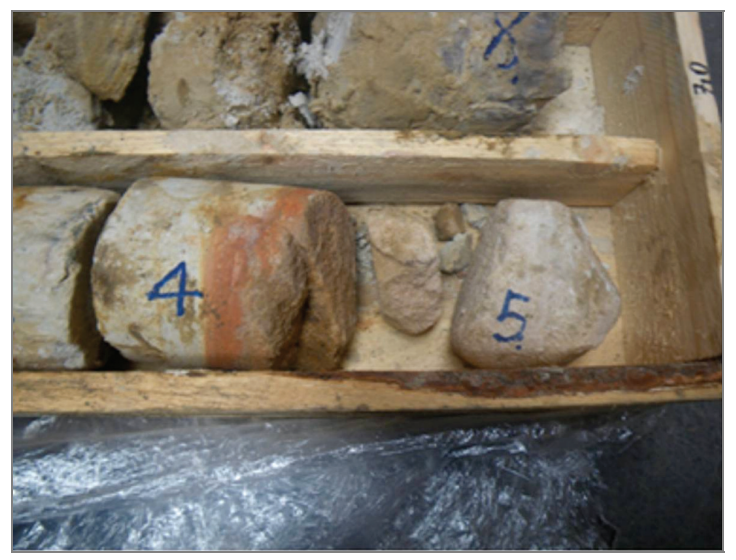

Fig. 1. Samples taken from the borehole located in Ruda Śląska (Silesia, Poland)
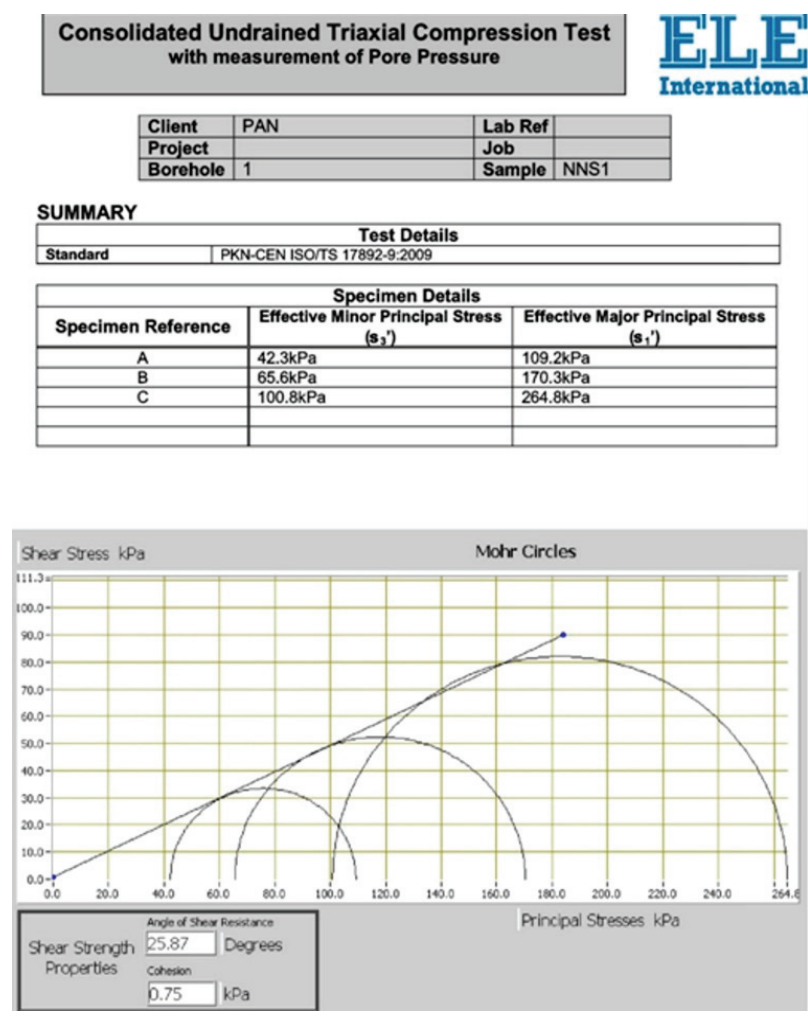

Fig. 2. Basic parameters of the Mohr-Coulomb material law determined on the basis of laboratory tests for geotechnical clay layer (depth: $-1.5 \div-2.9 \mathrm{~m}$ ) 
2. The analysis of the construction situation and technical condition of the building is carried out using the technical documentation, quantity surveys and a site inspection of the building. On this basis the information about the geometry, the loadcarrying structure, the types of joints, the materials used and the loads acting on the structure is obtained. If possible, samples of materials should be collected for laboratory tests. It is also important to analyse the type and depth of the foundations. This has an influence on determining the contact conditions existing on the interface between the soil and the foundations. Making an assumption that the interactions on the direction tangent to the contact surface are transferred through Coulomb friction, the value of the friction coefficient between the foundations and the soil should be determined. It depends both on the type of soil and the type of foundation surface.
The assessments of the building's resistance to the impact of mining, carried out for the purpose of planning the exploitation and usually conducted using a point method, can also be useful in assessing the technical condition [10]. An example of point-evaluation of six buildings and their category of resistance to the effects of subsoil deformation is shown in Fig. 3 [2].

3. The analysis of the mining situation is extremely important, both in terms of history and forecasts of exploitation. The forecasts provide information, for example, about the magnitude, direction and speed of the interactions. This information is the basis on which the boundary kinematic conditions are formulated in problems where the impact of the planned exploitation is considered. If the analysis concerns the assessment of the impact of ongoing or finished exploitation, these conditions are formulated on the basis of surveying measurements.

\begin{tabular}{|c|c|c|c|c|c|c|c|c|}
\hline \multirow[b]{2}{*}{ Ip. } & \multirow[b]{2}{*}{ features of the building } & \multirow[b]{2}{*}{ Feature (points) } & \multicolumn{6}{|c|}{ points for building } \\
\hline & & & $\begin{array}{c}1 \text { Maja } \\
228\end{array}$ & $\begin{array}{c}1 \text { Maja } \\
230\end{array}$ & $\begin{array}{c}1 \text { Maja } \\
232\end{array}$ & $\begin{array}{c}1 \text { Maja } \\
234\end{array}$ & $\begin{array}{c}1 \text { Maja } \\
236\end{array}$ & $\begin{array}{c}1 \text { Maja } \\
238\end{array}$ \\
\hline 1 & length of the building & $\begin{array}{l}\text { length } 15 \div 20 \mathrm{~m} \mathrm{(7)} \\
\text { length } 20 \div 30 \mathrm{~m} \mathrm{(15)}\end{array}$ & 15 & 15 & 15 & 15 & 7 & 15 \\
\hline 2 & shape of the building & $\begin{array}{l}\text { poorly fragmented cross-section, elongated solid (6) } \\
\text { cross-section of a simple, solid elongate (2) }\end{array}$ & 6 & 2 & 2 & 2 & 2 & 2 \\
\hline 3 & foundation of the building & $\begin{array}{l}\text { foundations at a constant level (0) } \\
\text { with no basement entrance gate ( } 8 \text { ) }\end{array}$ & 0 & 0 & 8 & 0 & 0 & 0 \\
\hline 4 & building base & rocky grounds with the exception of stony soils & 0 & 0 & 0 & 0 & 0 & 0 \\
\hline $5 a$ & foundations & $\begin{array}{l}\text { stony (4) } \\
\text { brick masonry (3) }\end{array}$ & 4 & 3 & 3 & 4 & 4 & 3 \\
\hline $5 b$ & basement walls & brick, stone or concrete blocks masonry & 3 & 3 & 3 & 3 & 3 & 3 \\
\hline $5 c$ & ceilings of lower floors & $\begin{array}{l}\frac{f}{l}<\frac{1}{10}(4) \\
\frac{f}{l}>\frac{1}{10}(2)\end{array}$ & 4 & 2 & 2 & 4 & 4 & 4 \\
\hline $5 d$ & lintels & $\begin{array}{l}\text { arched } \frac{f}{l}>\frac{1}{5}(3) \\
\text { reinforced concrete }(0) \\
\text { arched } \frac{f}{l}<\frac{1}{5}(5)\end{array}$ & 3 & 0 & 5 & 5 & 5 & 5 \\
\hline $5 e$ & other structural elements & & 0 & 0 & 0 & 0 & 0 & 0 \\
\hline 6 & existing protection & $\begin{array}{l}\text { no protection (15) } \\
\text { partial protection (12) }\end{array}$ & 15 & 15 & 15 & 15 & 12 & 15 \\
\hline $7 a$ & general technical condition & $\begin{array}{l}\text { inappropriate (3) } \\
\text { average (2) } \\
\text { satisfactory (1) }\end{array}$ & 2 & 1 & 2 & 2 & 1 & 3 \\
\hline $7 b$ & structural damage & $\begin{array}{l}\text { cracks to } 5 \mathrm{~mm}(5) \\
\text { scratches to } 1 \mathrm{~mm}(1) \\
\text { no damage (0) }\end{array}$ & 1 & 0 & 0 & 1 & 0 & 5 \\
\hline $8 a$ & factors that decrease & & 0 & 0 & 0 & 0 & 0 & 0 \\
\hline $8 b$ & factors increasing & & 0 & 0 & 0 & 0 & 0 & 0 \\
\hline & & total & 53 & 41 & 55 & 51 & 38 & 55 \\
\hline & cat & ory of resistance & 1 & 2 & 1 & 1 & 2 & 1 \\
\hline
\end{tabular}

Fig. 3. Point-evaluation of buildings and their category of resistance to the effects of mining subsoil deformation 


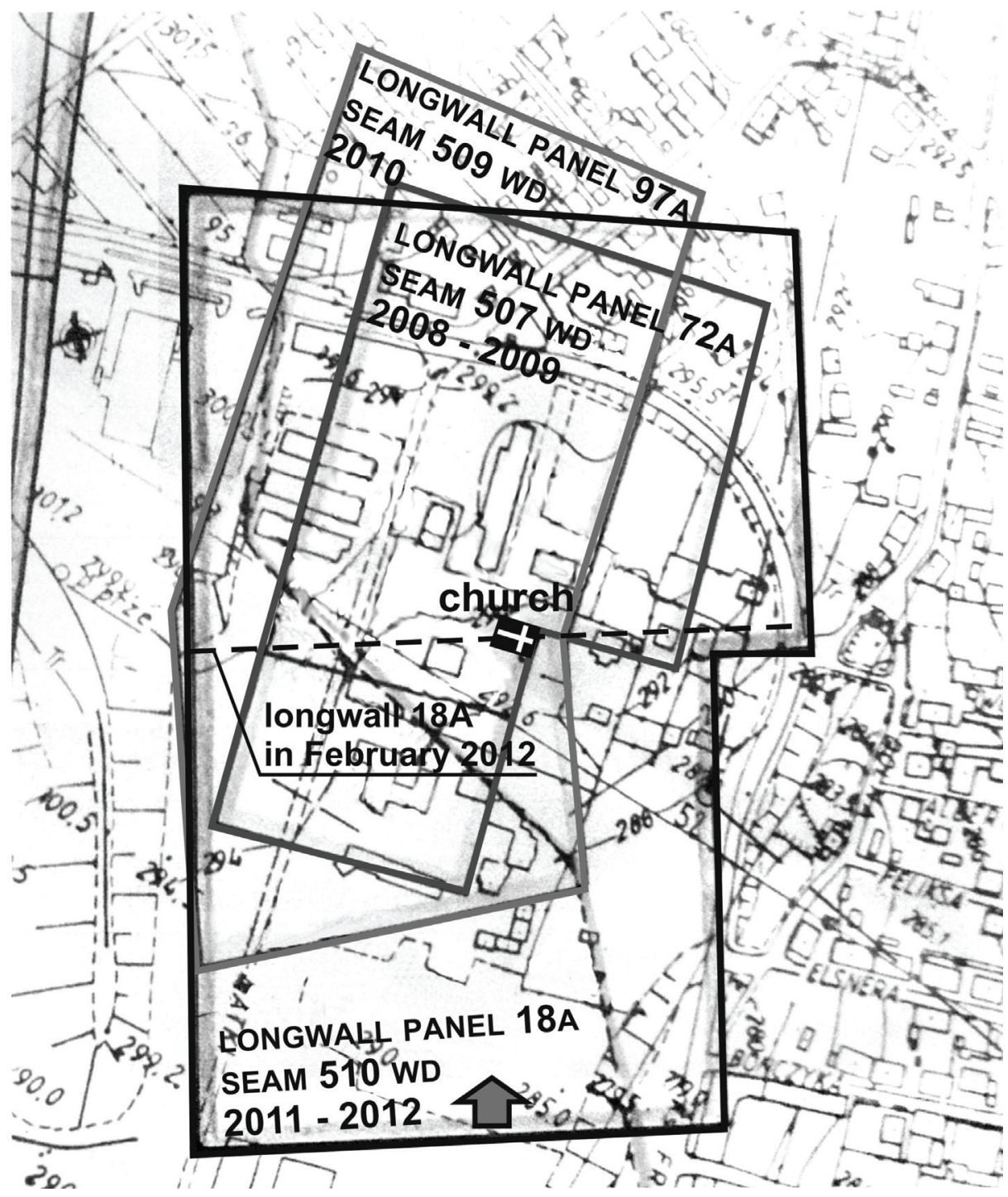

Fig. 4. Mining conditions of the sacred building located in Bytom (Silesia, Poland)

The analysis of the mining history of the rock mass is reflected in the assessment of the condition of the subsoil and of the probability of the occurrence of very negative effects of exploitation. Above all, these can be considerably greater values of subsoil deformation, caused by the disturbed structure of the rock mass or discontinuous deformations caused by the activation of old goaf or a negative overlap of the effects of exploitation in several fields. In areas where mining has been conducted for many years, the failure to include mining history in the analysis may have serious consequences. Figure 4 shows the image of a complicated mining situation of the sacral building (longwalls 72a and 97a), which influenced the occurrence of significant damage even before the manifestation of the influence of the longwall 18a excavation [6].
4. Laboratory tests of materials are done to determine the basic physical properties and to choose a constitutive law and determine its parameters. The extent of the tests, their type as well as the method of sample collection, sample size and number have a significant influence on the quality of this analysis. It must be remembered that, especially with weak soils, the destruction of the medium's structure during sample collection is very frequent. This in turn may lead to the results of laboratory experiments being far from reality. This risk is absent in the in situ methods of analysing deformability. However, the tests of this kind are quite costly, as a result of which they are used much less frequently. However, it might be expected that a situation arises when the geotechnical evaluation indicates that a test loading of the subsoil must be performed. If difficulties arise in collecting samples 
for laboratory tests to evaluate the mechanical parameters of the materials in existing buildings, nondestructive diagnostic methods can be used.

5. Surface deformation measurements. Surface deformations caused by mining are monitored by the mining company's surveyors by means of geodesic measurements conducted at scattered points or at points which form measurement lines. The documentation of these measurements is the source of information about the mining history of the area and the reaction of the rock mass to mining the deposit. These materials are used to evaluate the state of degradation of the rock mass, to predict the reaction of the area to the planned exploitation and to analyse the impact of finished exploitation on a building. In the last case the measurement results are used as a basis for the formulation of kinematic boundary condition. An example of surveying of surface deformation caused by longwall exploitation is shown in Fig. 5 [2].

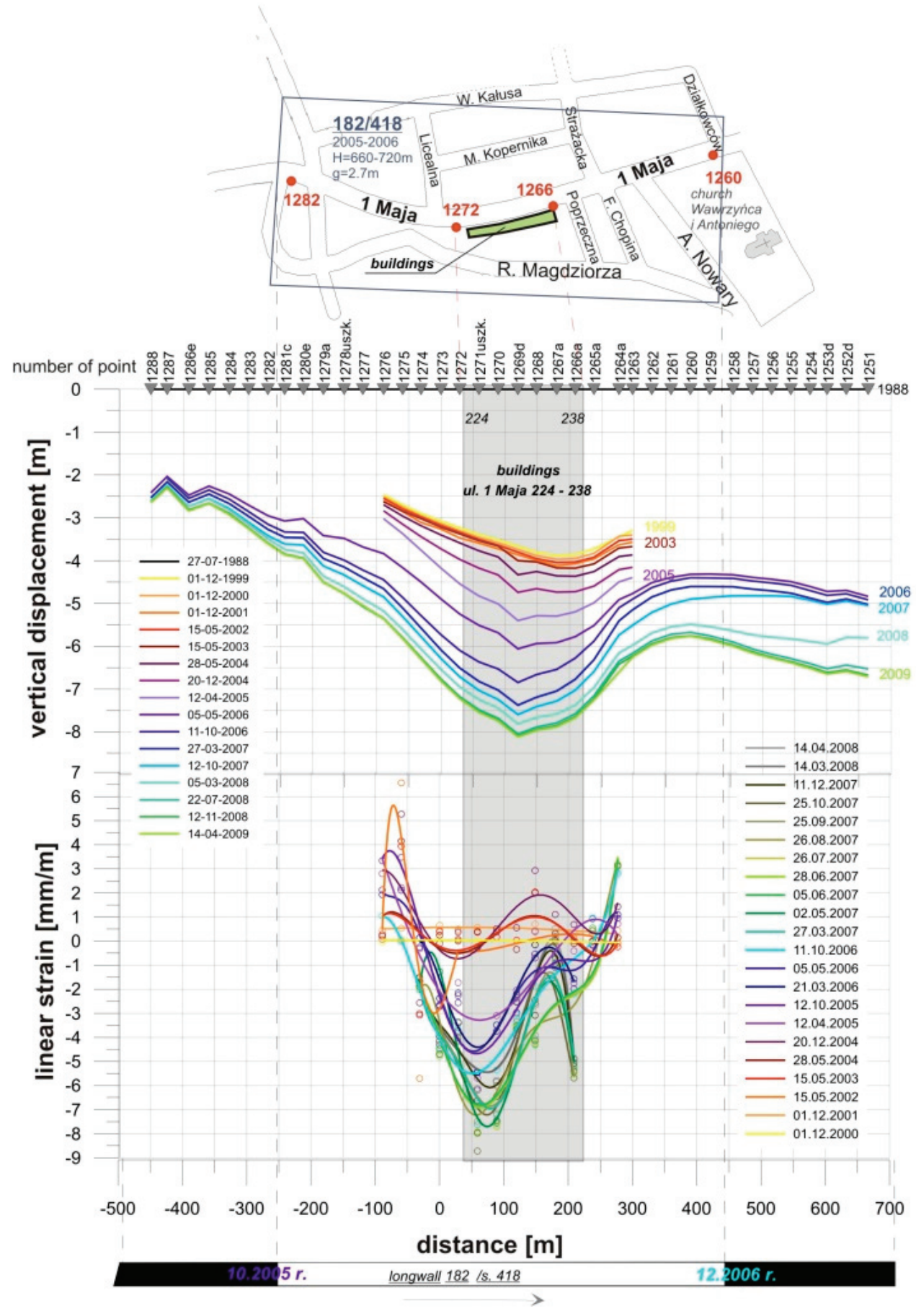

Fig. 5. Surveying of surface deformation caused by longwall exploitation 


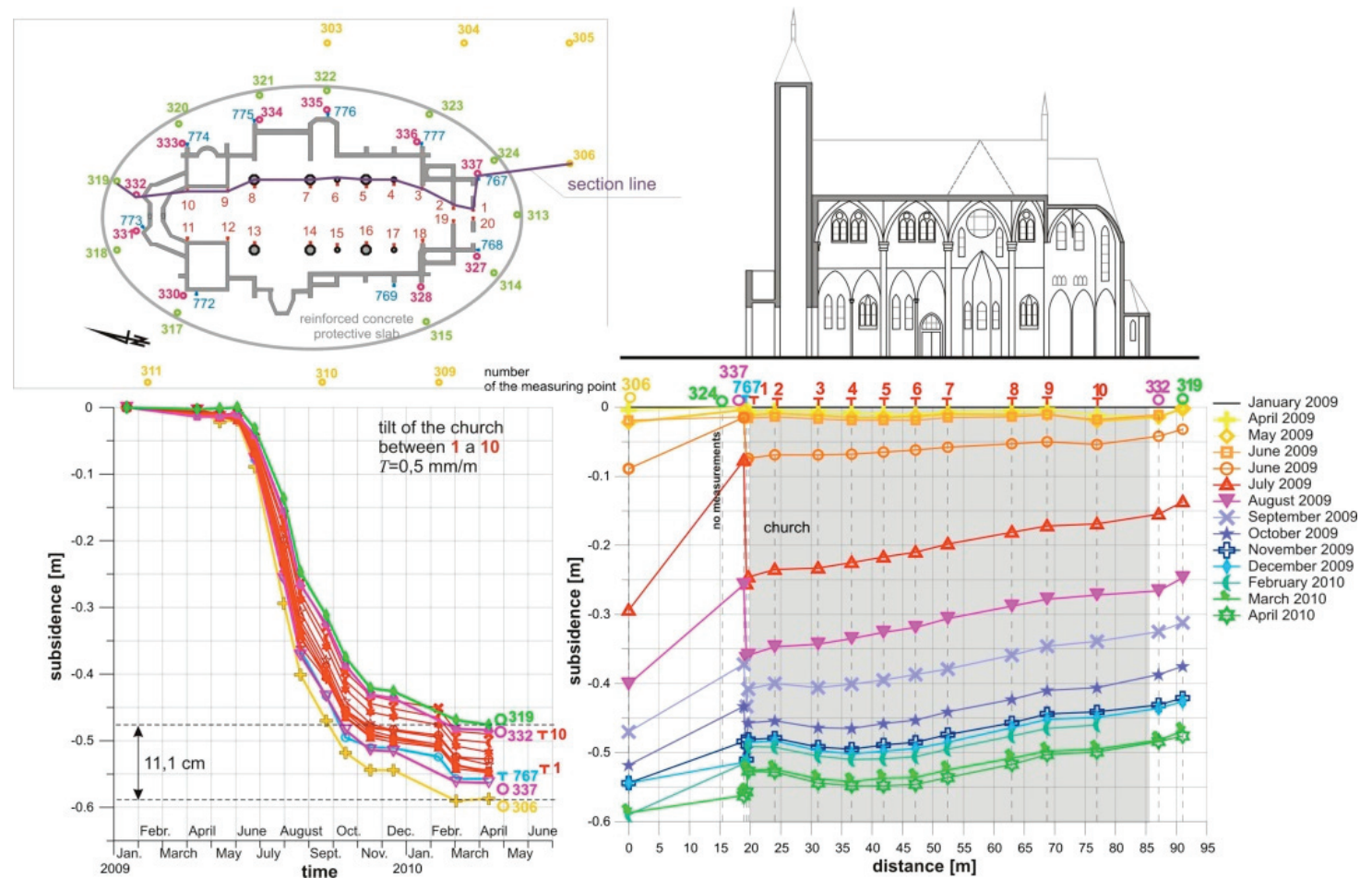

Fig. 6. The results of measurements of the deformations of buildings carried out using surveying methods. Subsidence measurements for the 306-319 cross-section for the church located in Ruda Śląska (Silesia, Poland)

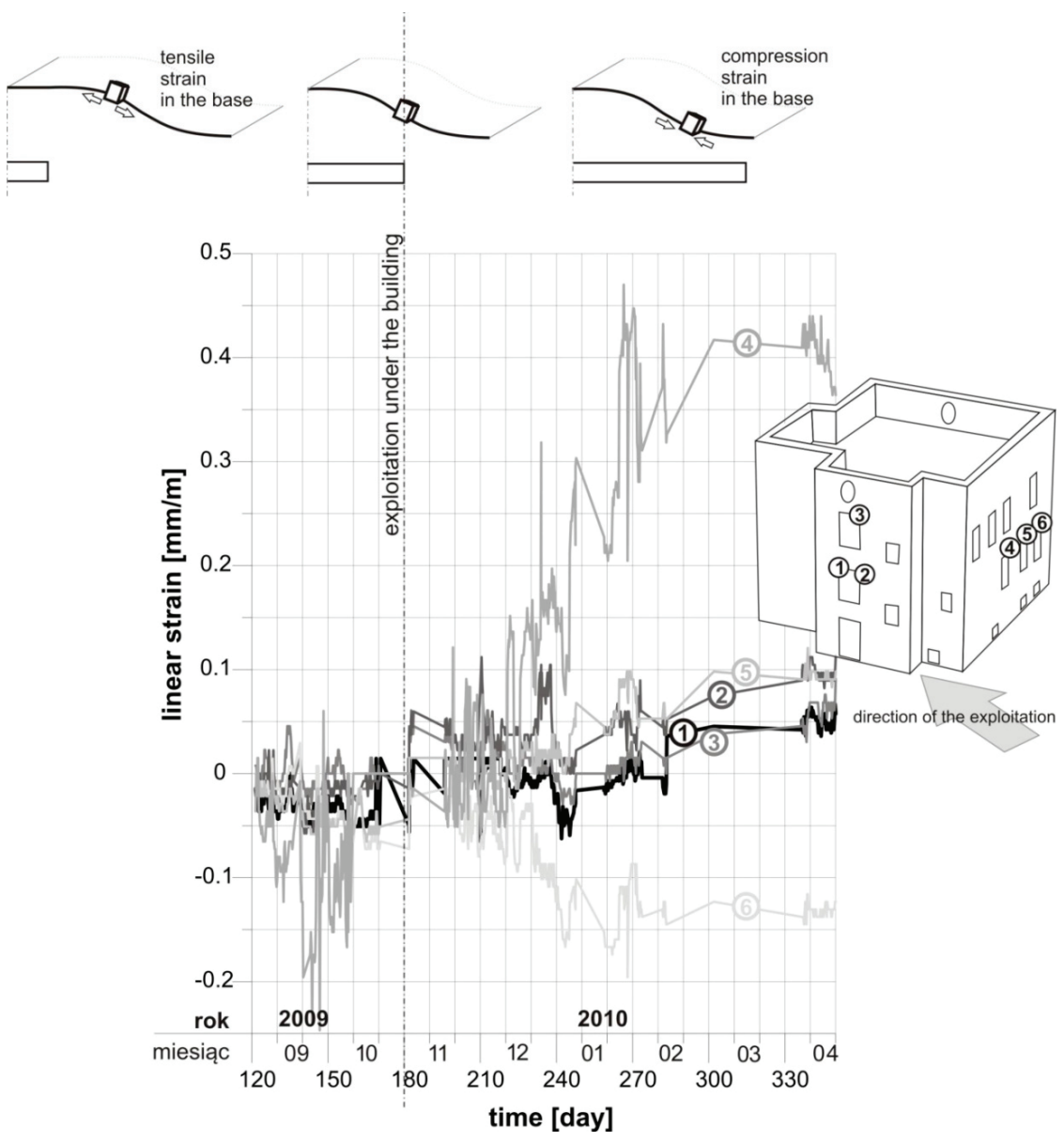

Fig. 7. The results of measurements of the deformations of buildings carried out using the automatic wireless monitoring system based on the extensometers 
6. Building deformation measurements. The measurement system installed on the building continuously monitors the condition of the building and can be used to validate the modelling process and to analyse the results obtained. Moreover, it is an interesting record of the building's reactions to the exploitation from a scientific point of view. Figures 6 and 7 show the results of measurements of the deformations of buildings carried out using surveying methods (Fig. 6) and using the automatic wireless monitoring system based on the extensometers (Fig. 7) [7], [5].

7. Calculation results analysis. Undoubtedly, the ability to appropriately interpret the results obtained from the calculations and measurements is an especially significant element. The solution to the mathematical problem provides an image of the state of stresses, strains and displacements of the entire building-subsoil system at any moment during exploitation. The results obtained should be subject to a reliable verification based on the knowledge and experience of the person conducting the analysis as well as the results of the observations and measurements of the deformation of the subsoil and the building.

Examples of numerical analyzes performed in accordance with the above guidelines are, among others, in the monograph of Florkowska (2010) [2].

\section{EXAMPLE OF NUMERICAL CALCULATIONS}

Figure 8 shows a scheme of the physical model as well as the FEM mesh model for which the analysis of
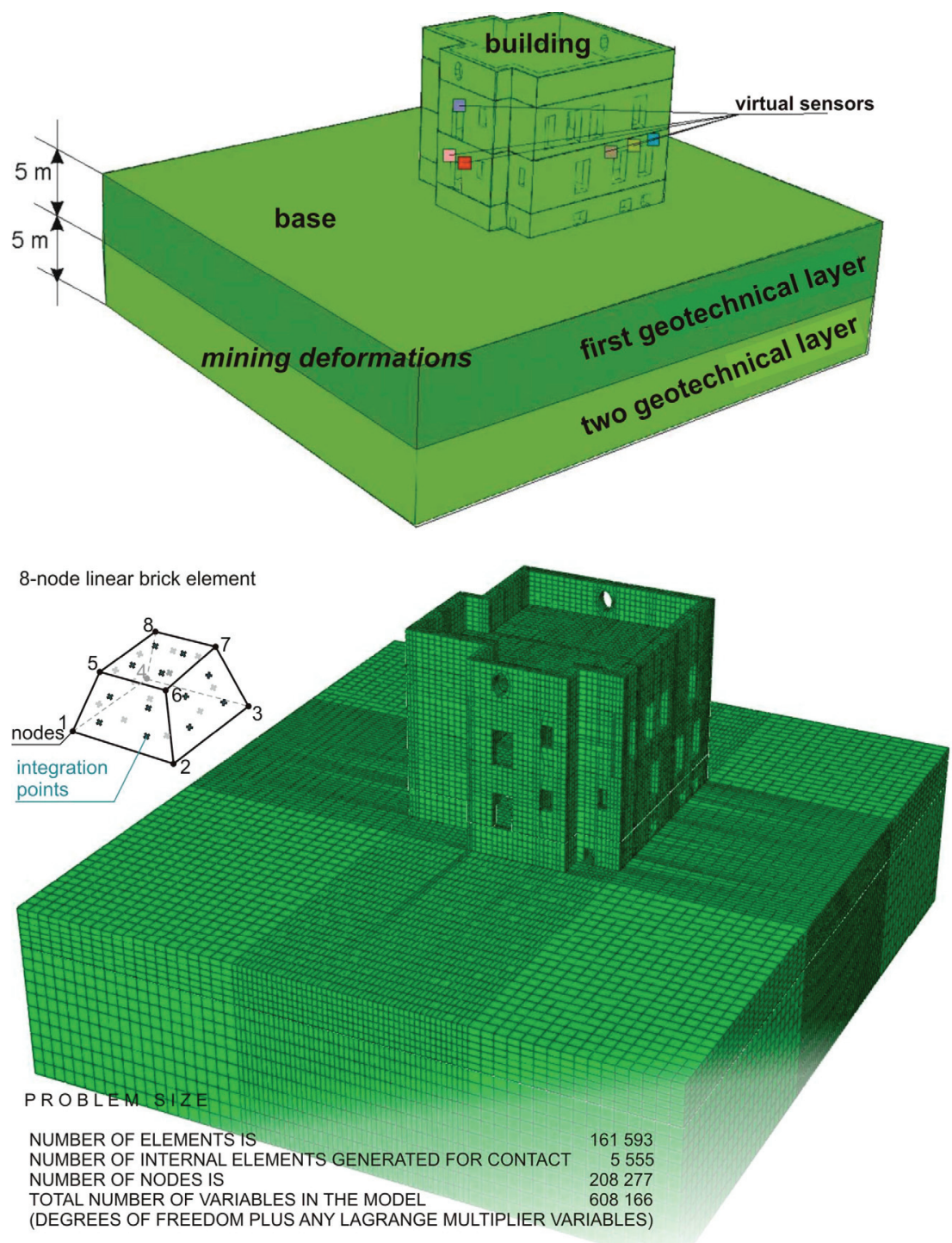

Fig. 8. The schematic physical model and the FEM mesh of the building and their base 

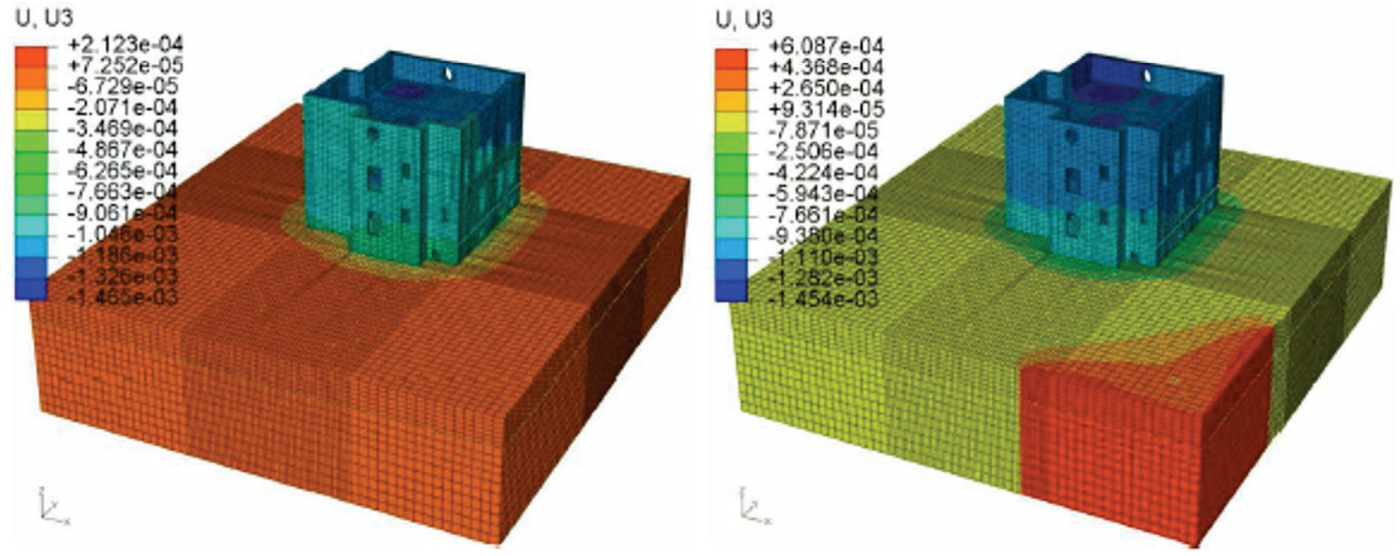

1

2
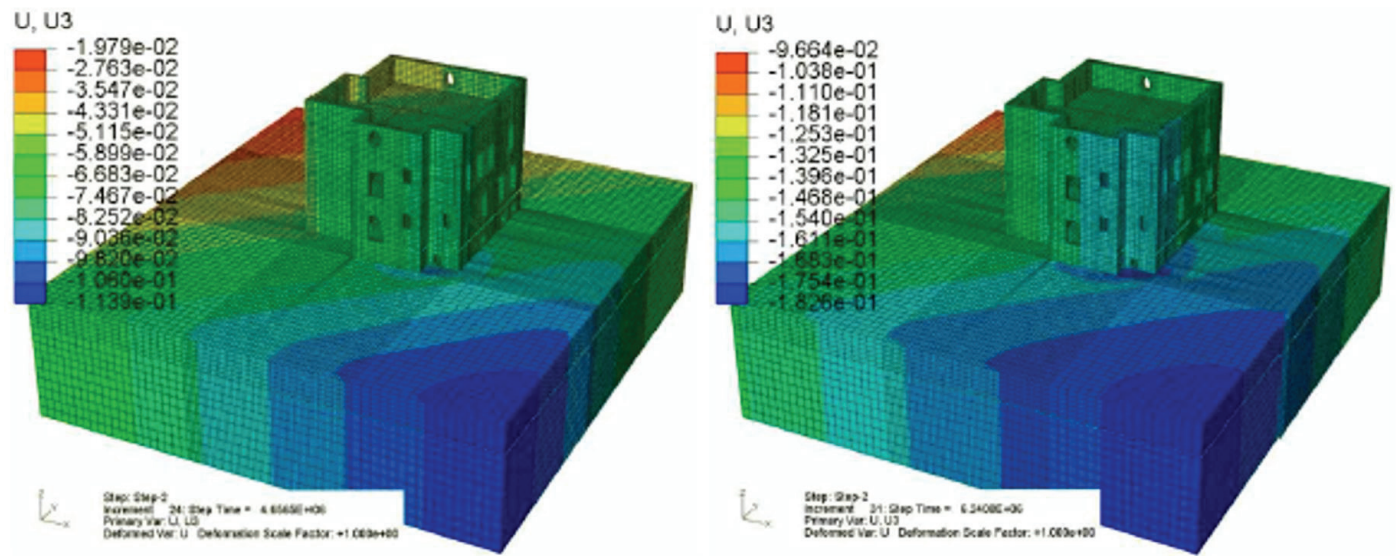

3

4

U, U3

U, U3
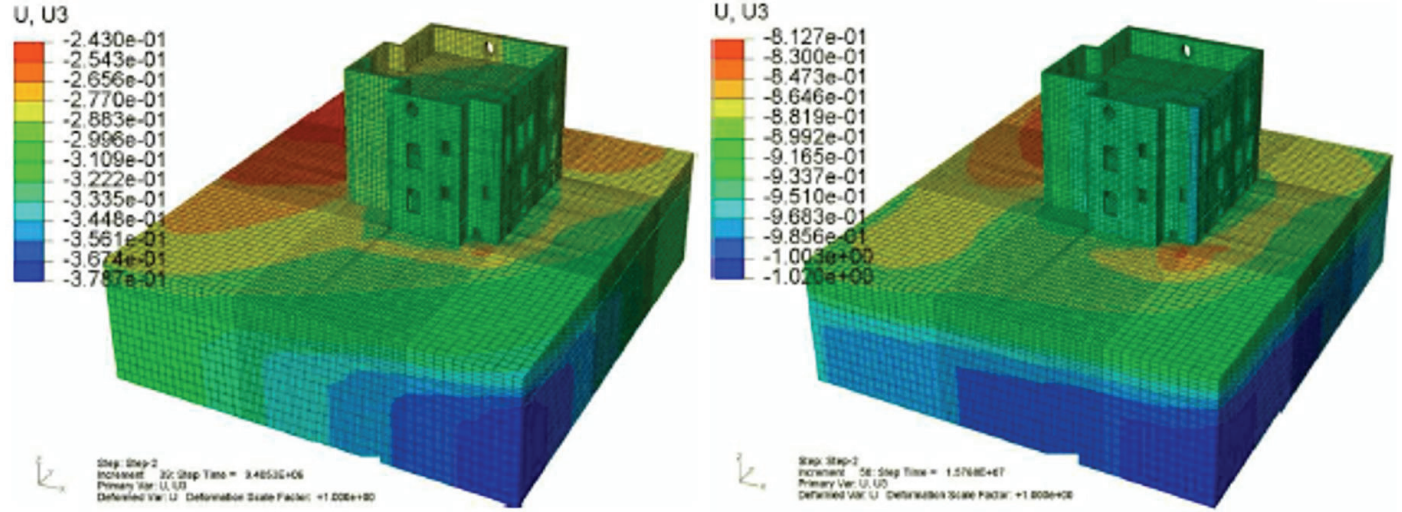

5

6

Fig. 9. Results of numerical calculations, which show a change in the state of vertical displacements of the building and its base on some subsequent steps of mining deformation

the impact of the planned mining exploitation, based on the prediction of mining deformation of the ground (according to the Budryk-Knothe theory), is performed. Calculations were performed using a model constructed on the basis of the mathematical description provided in Section 5. The results of numerical calculations, namely vertical displacements of the building and its base are presented in Fig. 9 [2]. The deformations are presented for some subsequent steps of mining deformation, numbered in the order from 1 to 6.

\section{SUMMARY}

In problems related to assessing the impact of underground mining on buildings, numerical modelling 
is practically the only method which makes it possible to carry out an analysis including all the factors which have a significant influence on the solution. However, there is no doubt that elaborating an appropriate model of this complex problem is a very difficult task.

This paper presents a synthetic collection of guidelines and hints on how to elaborate a mathematical model constituting a base of numerical analyses of this type of problems. Beginning with presenting the most important characteristics of the interacting building-deforming mining subsoil system, the authors formulate remarks first regarding the observation (which is the source of knowledge about the processes and phenomena which are elements of the problem) and then regarding the idealisation (as a process of selecting the significant characteristics which influence the solution). Then the authors offer a general mathematical formulation of the problem in the form of a contact problem of non-linear mechanics and suggest a numerical solution using the finite element method and applying the variational formulation using the virtual work principle.

An important part of the paper is a set of guidelines on how to properly determine input data, understood as a collection of information about the most important characteristics of the system and the results of the measurements on the basis of which the equations can be given specific forms and their parameters can be calculated.

The article is a brief compendium containing the basic information about the complex and difficult problems of numerical modelling of the impact of mining on buildings.

\section{REFERENCES}

[1] FlORKOwSKa L., Land subsidence due to Mining Operations in Disturbed Rock Mass, on the Example of Ruda Ślqska (Poland), Archives of Mining Sciences, 2010, Vol. 55, Iss. 3, 691-701.

[2] FlorkowsKa L., Application of numerical nonlinear mechanics in the issues of protecting buildings in mining areas (in Polish), Archives of Mining Sciences, Monografia, Nr 11, Kraków 2010.

[3] FLORKOWSKA L., Building protection against the backdrop of current situation and growth perspectives for polish mining industry, Archives of Mining Sciences, 2012, Vol. 57, Iss. 3, $655-665$

[4] FlorkowsKa L., Numerical modelling for underground mining related geotechnical issues, Studia Geotechnica et Mechanica, 2013, No. 3, 13-24.

[5] FLORKOWSKA L., Remote monitoring of strain in the building caused by underground mining exploitation. Example of implementation, (in Polish), Inżynieria i Budownictwo, 2013, No. 3, 154-156.

[6] Florkowska L., KanciruK A., Analysis of the consequences of mining exploitation in substantially disturbed strata based on spatial measurements of a building's tilt, Rock Mechanics for Resources, EUROCK 2013. CRC Press. 575-580.

[7] FloRKOWSKa L., Analysis of the impact of longwall mining on sacred historic building, Architecture - Civil Engineering - Environment - ACEE, 2013, No. 2, 15-27.

[8] KNOTHE S., Equation of subsiding trough's curvature (in Polish), Archiwum Górnictwa i Hutnictwa, 1953, T. 1, Zeszyt 1, 111-127.

[9] KNOTHE S., Time influence on a formation of a subsidence through (in polish), Archiwum Górnictwa i Hutnictwa, 1953, T. 1, Zeszyt 1, 128-139.

[10] WiTAKOWSKi P., Wireless monitoring of buildings, (in Polish), Materials IX Symposium "System of Total Quality Management in Civil Engineering”, ITB, Warszawa 2010. 